Article

\title{
The Effect of Sodium-Dependent Glucose Cotransporter 2 Inhibitor Tofogliflozin on Neurovascular Coupling in the Retina in Type 2 Diabetic Mice
}

\author{
Junya Hanaguri ${ }^{1}$, Harumasa Yokota ${ }^{1} \mathbb{D}$, Akifumi Kushiyama ${ }^{2} \mathbb{D}$, Sakura Kushiyama ${ }^{3}$, Masahisa Watanabe ${ }^{1}$, \\ Satoru Yamagami ${ }^{1}$ and Taiji Nagaoka ${ }^{1, *}$
}

Citation: Hanaguri, J.; Yokota, H.; Kushiyama, A.; Kushiyama, S.;

Watanabe, M.; Yamagami, S.;

Nagaoka, T. The Effect of

Sodium-Dependent Glucose

Cotransporter 2 Inhibitor

Tofogliflozin on Neurovascular

Coupling in the Retina in Type 2

Diabetic Mice. Int. J. Mol. Sci. 2022,

23, 1362. https://doi.org/10.3390/ ijms23031362

Academic Editor: Settimio Rossi

Received: 14 December 2021

Accepted: 19 January 2022

Published: 25 January 2022

Publisher's Note: MDPI stays neutral with regard to jurisdictional claims in published maps and institutional affiliations.

Copyright: (C) 2022 by the authors. Licensee MDPI, Basel, Switzerland. This article is an open access article distributed under the terms and conditions of the Creative Commons Attribution (CC BY) license (https:// creativecommons.org/licenses/by/ $4.0 /)$.
1 Division of Ophthalmology, Department of Visual Science, Nihon University School of Medicine, Itabashi, Tokyo 173-8601, Japan; locksteady@icloud.com (J.H.); atokoy18@gmail.com (H.Y.); masahisa_watanabe12-1@yahoo.co.jp (M.W.); yamagami.satoru@nihon-u.ac.jp (S.Y.)

2 Department of Pharmacotherapy, Meiji Pharmaceutical University, Kiyose, Tokyo 204-8588, Japan; kushiyama@my-pharm.ac.jp

3 Division of Life Science, Department of Nursing, National College of Nursing, Kiyose, Tokyo 204-8575, Japan; kushiyamake@gmail.com

* Correspondence: taijinagaoka@gmail.com; Tel.: +81-3-3972-8111

\begin{abstract}
We investigated the effect of tofogliflozin, a sodium-dependent glucose cotransporter 2 inhibitor (SGLT2i), on retinal blood flow dysregulation, neural retinal dysfunction, and the impaired neurovascular coupling in type 2 diabetic mice. Tofogliflozin was added to mouse chow to deliver $5 \mathrm{mg} / \mathrm{kg} /$ day and 6-week-old mice were fed for 8 weeks. The longitudinal changes in the retinal neuronal function and blood flow responses to systemic hyperoxia and flicker stimulation were evaluated every 2 weeks in diabetic $\mathrm{db} / \mathrm{db}$ mice that received tofogliflozin $(n=6)$ or placebo $(n=6)$ from 8 to 14 weeks of age. We also evaluated glial activation and vascular endothelial growth factor (VEGF) expression by immunofluorescence. Tofogliflozin treatment caused a sustained decrease in blood glucose in $\mathrm{db} / \mathrm{db}$ mice from 8 weeks of the treatment. In tofogliflozin-treated $\mathrm{db} / \mathrm{db}$ mice, both responses improved from 8 to 14 weeks of age, compared with vehicle-treated diabetic mice. Subsequently, the electroretinography implicit time for the oscillatory potential was significantly improved in SGLT2i-treated $\mathrm{db} / \mathrm{db}$ mice. The systemic tofogliflozin treatment prevented the activation of glial fibrillary acidic protein and VEGF protein expression, as detected by immunofluorescence. Our results suggest that glycemic control with tofogliflozin significantly improved the impaired retinal neurovascular coupling in type 2 diabetic mice with the inhibition of retinal glial activation.
\end{abstract}

Keywords: diabetic retinopathy; neurovascular coupling; SGLT2 inhibitor; glial cell function; microvascular complication

\section{Introduction}

Diabetic retinopathy continues to be a leading cause of blindness [1]. The prospective observational clinical study with type 2 diabetes revealed that hemoglobin A1c was the only factor associated with both the incidence and progression of diabetic retinopathy (DR) [2], suggesting that blood glucose levels are very important to prevent the DR in type 2 diabetes. Therefore, glycemic control is the most important goal after the onset of diabetes with no apparent retinopathy.

Sodium-glucose cotransporter 2 (SGLT2) inhibitors (SGLT2i) are a new class of oral anti-diabetic agent that reduces the blood glucose level by inhibiting glucose reabsorption in the proximal renal tubules. These agents are commonly used by young obese patients with type 2 diabetes because weight reduction can be expected as calories are lost when glucose is excreted in the urine [3]. Recently, some clinical case series have shown the beneficial effects of SGLT2i on DR [4,5] and diabetic macular edema [6,7]. However, the EMPA-REG 
outcome trial could not identify a beneficial effect of empagliflozin on DR [8]. Moreover, no study has examined the effect of SGLT2i on retinal function in diabetic animals.

Our recent study revealed that retinal blood flow (RBF) does not respond to systemic hyperoxia and flicker stimulations in $\mathrm{db} / \mathrm{db}$ mice with early type 2 diabetes, and no apparent changes in resting retinal perfusion were observed [9]. These results suggest that long-term glucotoxicity is a central mechanism for impaired retinal neurovascular coupling in type 2 diabetic mice. Glucose lowering by the early detection of RBF dysregulation and prompt treatment may, therefore, protect retinal tissue and prevent or slow the development of irreversible retinopathy. We investigated the effect of long-term (8 weeks) systemic treatment with tofogliflozin, an SGLT2 inhibitor, on RBF dysregulation and neural retinal dysfunction in type 2 diabetic mice.

\section{Results}

\subsection{The Effect of the Longitudinal Administration of Tofogliflozin on Glycemic Control}

In this study, the drug treatment of $\mathrm{db} / \mathrm{db}$ mice was initiated from 6 weeks of age. Blood glucose was measured every 2 weeks from 6 weeks before the start of treatment to 14 weeks of age. The casual blood glucose levels in $\mathrm{db} / \mathrm{db}$ mice treated with tofogliflozin $(n=6)$ significantly decreased in comparison with untreated $\mathrm{db} / \mathrm{db}$ mice $(n=6)$ from 8 to 14 weeks. At 14 weeks of age, the blood glucose levels were untreated and SGLT2i-treated $\mathrm{db} / \mathrm{db}$ mice were $440.2 \pm 54.0$ and $262.2 \pm 43.3 \mathrm{mg} / \mathrm{dL}$, respectively (Table 1 ).

Table 1. Longitudinal changes in casual blood glucose (mg/dL).

\begin{tabular}{cccccc}
\hline Age (Weeks) & $\mathbf{6}$ & $\mathbf{8}$ & $\mathbf{1 0}$ & $\mathbf{1 2}$ & $\mathbf{1 4}$ \\
\hline $\mathrm{db} / \mathrm{db}+$ normal feed $(n=6)$ & $340.0 \pm 41.8$ & $419.0 \pm 41.3$ & $351.8 \pm 19.8$ & $331.5 \pm 13.8$ & $440.2 \pm 54.0$ \\
\hline $\mathrm{db} / \mathrm{db}+$ Tofogliflozine $(n=6)$ & $310.7 \pm 30.0$ & $226.0 \pm 19.1^{*}$ & $232.0 \pm 32.4^{*}$ & $202.5 \pm 20.5^{*}$ & $264.2 \pm 43.3^{*}$ \\
\hline
\end{tabular}

Data are expressed as the mean \pm standard error of the mean (SEM). ${ }^{*} p<0.05 \mathrm{vs} . \mathrm{db} / \mathrm{db}+$ normal feed. These agents are commonly used by young obese patients with type 2 diabetes because weight reduction can be expected as calories are lost when glucose is excreted in the urine [3].

\subsection{Longitudinal Changes of Systemic and Ocular Parameters}

During the experiment, there were no significant differences in body weight, systemic blood pressure (BP), intraocular pressure (IOP), or ocular perfusion pressure (OPP) between treated and untreated mice (Figure 1).

\subsection{Longitudinal Assessment of Resting RBF in Diabetic Mice}

The resting RBF is shown in Figure 2. Both untreated $\mathrm{db} / \mathrm{db}$ and treated $\mathrm{db} / \mathrm{db}$ showed a steady resting RBF from 8 to 14 weeks of age, with no significant difference between the groups (two-way repeated-measures analysis of variance (ANOVA)).

\subsection{Longitudinal Assessment of RBF in Response to Systemic Hyperoxia in Diabetic Mice}

In 8-week-old $\mathrm{db} / \mathrm{db}$ mice, a significant difference in the hyperoxia-induced change in RBF was observed between the treatment groups (two-way repeated-measures ANOVA; Figure 3A). The significant differences in hyperoxia-induced flow also were observed at 10 , 12 , and 14 weeks of age (Figure 3B-D).

\subsection{Longitudinal Assessment of RBF in Response to Flicker Stimulation in Diabetic Mice}

The temporal change in RBF in response to a 3 min light flicker stimulation was evaluated on experimental day 2 . In 8 -week-old $\mathrm{db} / \mathrm{db}$ mice, there was a significant difference in the flicker-induced change in RBF between untreated and treated mice (two-way repeatedmeasures ANOVA; Figure 4A). Significant differences in flicker-induced flow changes also were at 10, 12, and 14 weeks of age (Figure $4 \mathrm{~B}-\mathrm{D})$. 
A

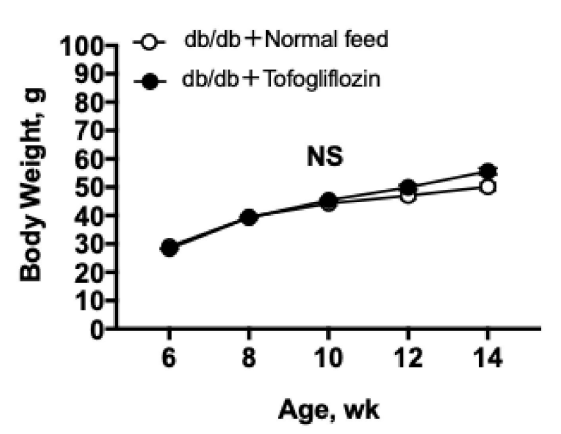

D

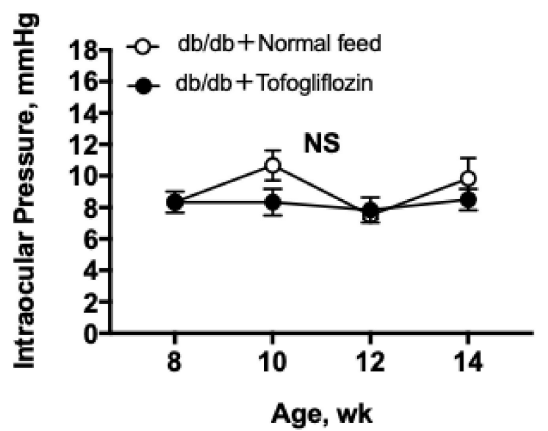

B

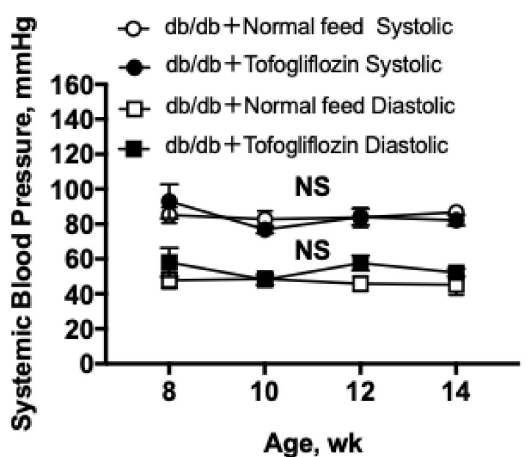

E

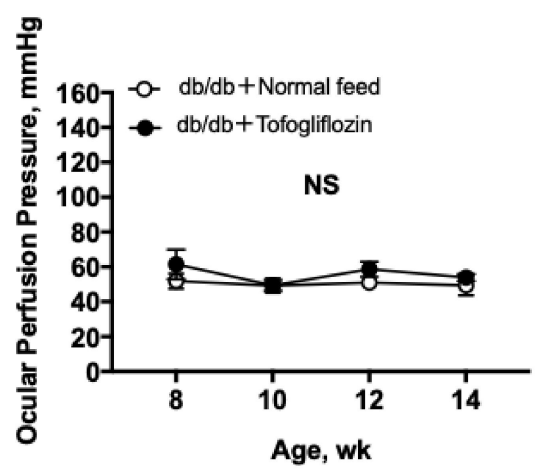

C

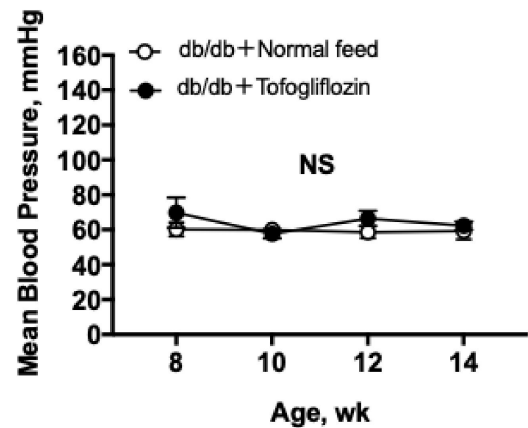

Figure 1. Tofogliflozin did not change the level of other systemic or ocular parameters: (A) body weight; (B) systemic BP; (C) mean BP; (D) IOP; (E) OPP. Body weight was measured every 2 weeks from 8 weeks to 14 weeks of age. Systemic BP, mean BP, IOP, and OPP were measured every 2 weeks from 8 weeks to 14 weeks of age. Each parameter was compared between tofogliflozin-treated $\mathrm{db} / \mathrm{db}$ mice $(\mathrm{db} / \mathrm{db}+$ tofogliflozin) $(n=6)$ in $\mathrm{db} / \mathrm{db}$ mice fed normal chow $(\mathrm{db} / \mathrm{db}+$ normal feed $)(n=6)$ by two-way repeated-measures analysis of variance (ANOVA). Data are expressed as the mean \pm SEM. NS indicates no significant difference between groups.

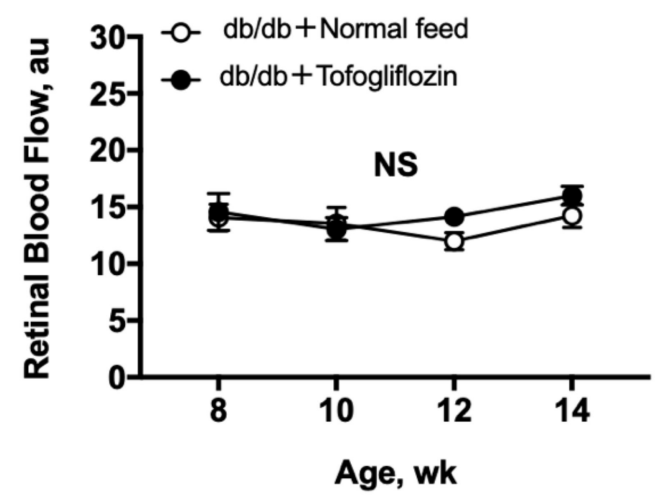

Figure 2. Longitudinal resting RBF remains stable throughout the measurement period from 8 to 14 weeks of age in $\mathrm{db} / \mathrm{db}$ mice and were not different for $\mathrm{db} / \mathrm{db}$ mice treated with tofogliflozin $(n=6)$ and $\mathrm{db} / \mathrm{db}$ mice fed normal chow $(n=6)$ from 8 to 14 weeks of age. RBF was measured every 2 weeks, and the statistical analyses of the results of each treatment over time were performed by a one-way repeated measures ANOVA $(p=0.32$ for $\mathrm{db} / \mathrm{db}+$ normal feed mice and $p=0.34$ for $\mathrm{db} / \mathrm{db}+$ tofogliflozin mice). The differences between 2 weeks in the resting RBF were tested in the 2 groups by a two-way repeated-measures ANOVA $(p=0.24)$. au indicates arbitrary unit; NS indicates no significant difference between groups and within a group. Data are expressed as the mean \pm SEM. 
A

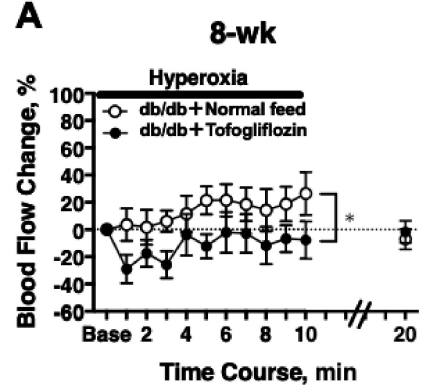

C

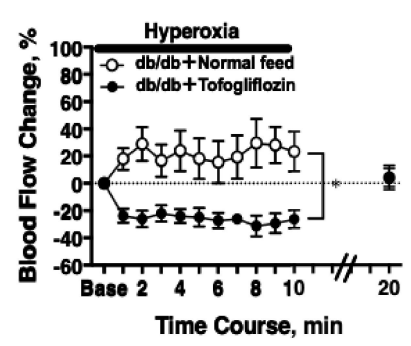

B

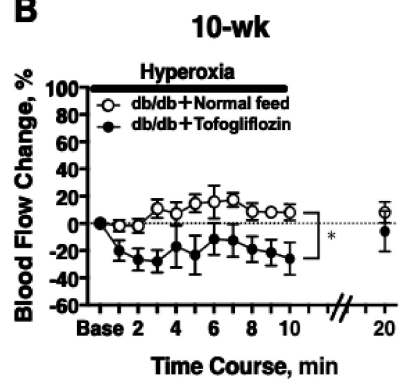

D

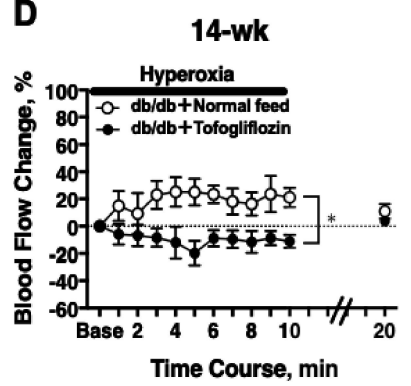

Figure 3. The RBF response to systemic hyperoxia was longitudinally retained in $\mathrm{db} / \mathrm{db}$ mice treated with tofogliflozin ( $\mathrm{db} / \mathrm{db}+$ tofogliflozin, $n=6)$ and blunted in $\mathrm{db} / \mathrm{db}$ mice fed normal chow $(\mathrm{db} / \mathrm{db}+$ normal feed, $n=6)$ from 8 to 14 weeks of age: (A) 8 weeks of age; (B) 10 weeks of age; (C) 12 weeks of age; (D) 14 weeks of age. ${ }^{*} p<0.05$ between groups analyzed by repeated-measures ANOVA; solid bar = period of hyperoxia.

A

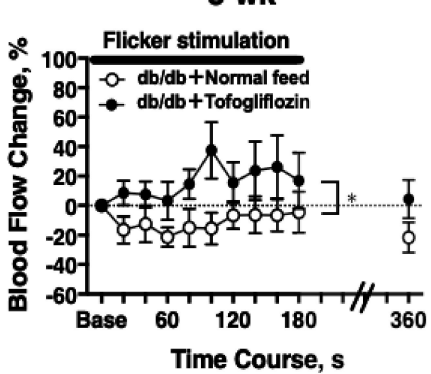

C

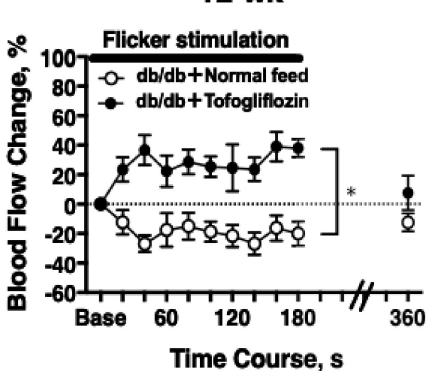

B

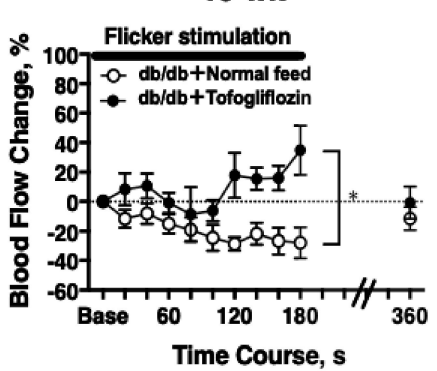

D

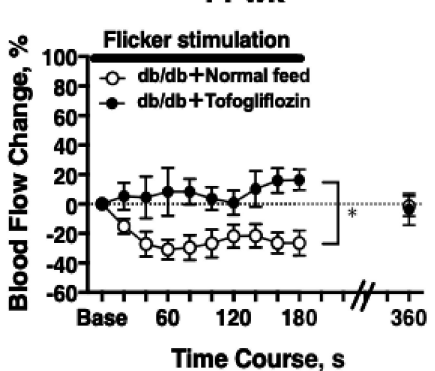

Figure 4. The RBF response to flicker stimulation was longitudinally retained in $\mathrm{db} / \mathrm{db}$ mice treated with tofogliflozin ( $\mathrm{db} / \mathrm{db}+$ tofogliflozin, $n=6)$ and blunted in $\mathrm{db} / \mathrm{db}$ mice fed normal chow ( $\mathrm{db} / \mathrm{db}+$ normal feed, $n=6)$ from 8 to 14 weeks of age: (A) 8 weeks of age; (B) 10 weeks of age; (C) 12 weeks of age; (D) 14 weeks of age. ${ }^{*} p<0.05$ between groups analyzed by a repeated-measures ANOVA; solid bar $=$ period of flicker stimulation.

\subsection{Longitudinal Assessment of Electroretinography (ERG) Parameters}

The amplitude or implicit time of the a-wave and b-wave on ERG in treated and untreated $\mathrm{db} / \mathrm{db}$ mice showed no significant changes with age (Figure $5 \mathrm{~A}-\mathrm{D}$; one-way 
and two-way repeated-measures ANOVA). While no significant differences were found between treated and untreated $\mathrm{db} / \mathrm{db}$ mice in the implicit time of OP3 (Figure 5G) and the $\Sigma \mathrm{OP}$ amplitude (Figure 5H), the implicit times of OP1 and OP2 in SGLT2i tofogliflozintreated $\mathrm{db} / \mathrm{db}$ mice decreased significantly, compared with those in untreated $\mathrm{db} / \mathrm{db}$ mice from 12 to 14 weeks of age (Figure 5E; two-way repeated-measures ANOVA).

A

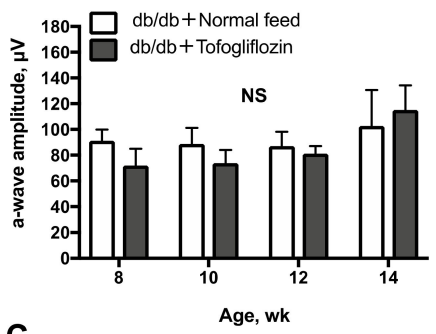

C

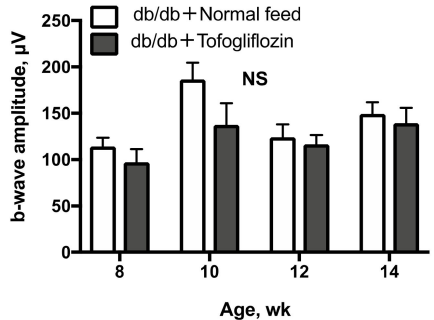

E

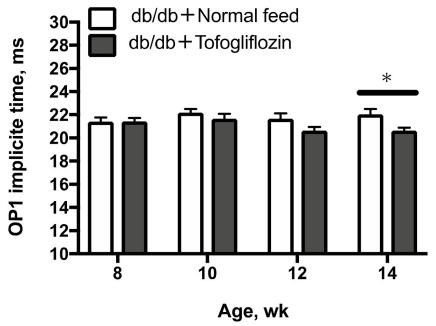

G

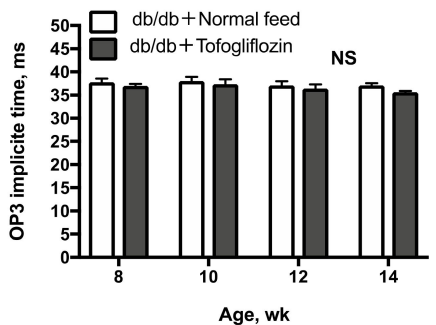

B

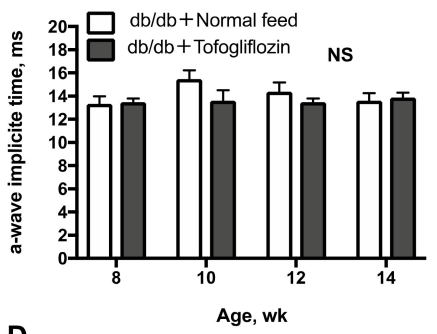

D

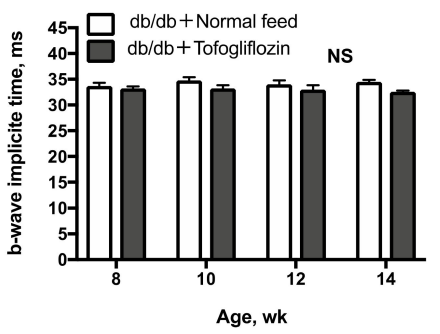

F

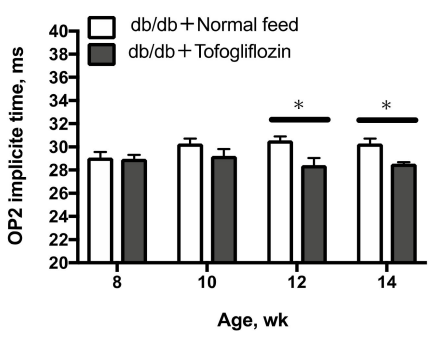

$\mathbf{H}$

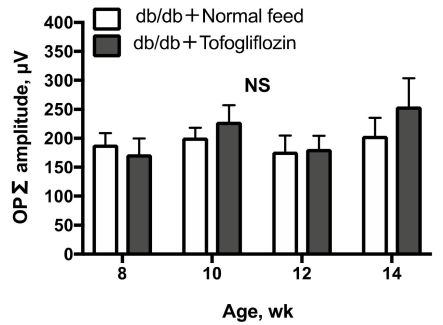

Figure 5. Longitudinal assessment of ERGs in $\mathrm{db} / \mathrm{db}$ mice treated with tofogliflozin $(\mathrm{db} / \mathrm{db}+$ tofogliflozin, $n=6$ ) and blunted in $\mathrm{db} / \mathrm{db}$ mice fed normal chow ( $\mathrm{db} / \mathrm{db}+$ normal feed, $n=6)$ from 8 to 14 weeks of age: (A) amplitude of the a-wave; (B) implicit time of the a-wave; (C) amplitude of the b-wave; (D) implicit time of the b-wave; (E) implicit time of OP1 waves; (F) implicit time of OP2 waves; (G) implicit time of OP3 waves; $(\mathbf{H})$ total amplitudes of OP waves $(\Sigma \mathrm{OP})$. There were no significant differences in the implicit time or amplitude of the a-waves $(\mathbf{A}, \mathbf{B})$ and b-waves $(\mathbf{C}, \mathbf{D})$ between the $\mathrm{db} / \mathrm{db}+$ tofogliflozin mice and $\mathrm{db} / \mathrm{db}+$ normal feed mice. There were no significant changes in the implicit time of the OP3 (G) waves or the total amplitudes of the OP waves ( $\mathrm{OPP})$ (H) between the groups of mice (two-way repeated-measures ANOVA). The implicit time of OP1 (E) increased significantly in $\mathrm{db} / \mathrm{db}+$ normal feed mice at 14 weeks of age, and the implicit time of the OP2 waves $(\mathbf{F})$ increased significantly in $\mathrm{db} / \mathrm{db}+$ normal feed mice from 12 to 14 weeks of age (two-way repeated-measures ANOVA). ${ }^{*} p<0.05$, between groups. 
2.7. Comparison of the Maximal Change in RBF in Response to Hyperoxia and Flicker Stimulation at 14 Weeks of Age in Diabetic Mice and db/m Non-Diabetic Control Mice

At 14 weeks of age, the same protocol for systemic hyperoxia and flicker stimulation was performed in two diabetic mice and $\mathrm{db} / \mathrm{m}$ control mice groups (Figure 6 ). We previously confirmed that the $\mathrm{RBF}$ response to both stimulations remained stable in $\mathrm{db} / \mathrm{m}$ non-diabetic control mice from 8 to 20 weeks of age [9]. RBF measurements were performed only once at only 14 weeks of age in line with animal welfare criteria for our institution. Figure 6 shows the changes in RBF in response to both systemic hyperoxia (Figure 6A) and flicker stimulation (Figure 6B). In Figure 6, the changes observed in control $\mathrm{db} / \mathrm{m}$ mice were not present in the untreated $\mathrm{db} / \mathrm{db}$ mice; however, in the SGLT2i tofogliflozin group, these changes were approximately half of those in the $\mathrm{db} / \mathrm{m}$ control group.

(A) Systemic Hyperoxia

\section{Maximum Response of Retinal Blood Flow, \%}

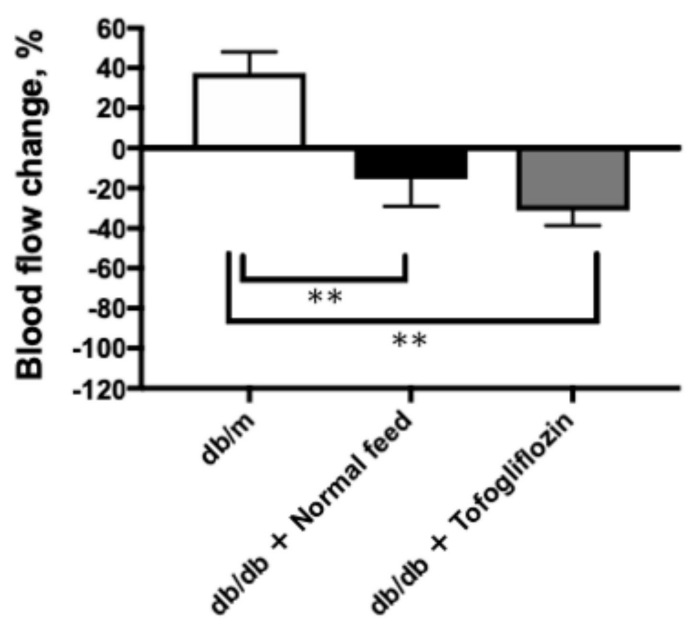

(B) Flicker Stimulation

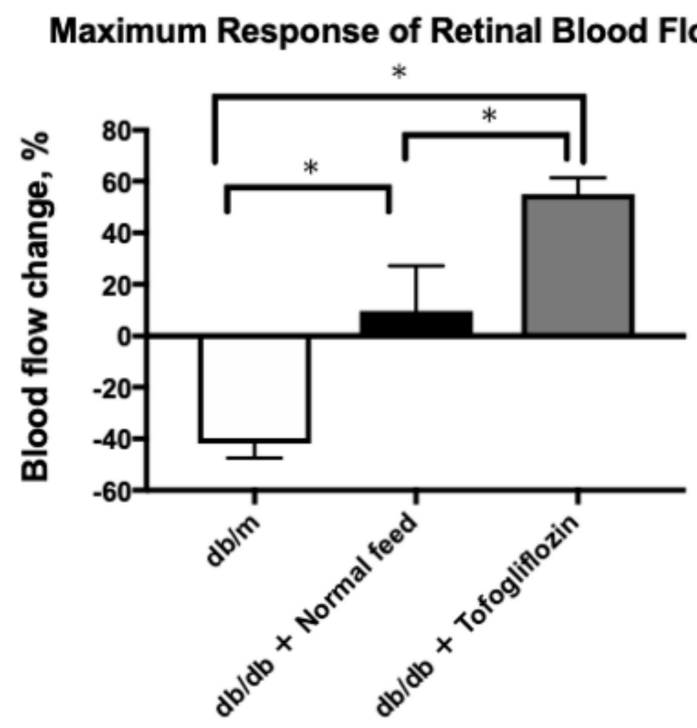

Figure 6. Maximal changes in RBF from baseline in response to hyperoxia (A) and flicker stimulation (B) in 14-week-old mice of $\mathrm{db} / \mathrm{m}(n=5)$ as a non-diabetic control and untreated $\mathrm{db} / \mathrm{db}$ mice $(n=6)$ and tofogliflozin-treated diabetic mice $(n=6) .{ }^{*} p<0.05,{ }^{* *} p<0.01$ compared with $\mathrm{db} / \mathrm{m}$ by a one-way ANOVA followed by Holm-Sidak test.

\subsection{Beneficial Effect of the Systemic Administration of Tofogliflozin on Glial Fibrillary Acidic} Protein (GFAP) and VEGF Expression

To clarify the beneficial effect of tofogliflozin on the pathogenesis of DR, we performed immunohistochemical analysis to investigate whether the long-term systemic administration of SGLT2 $i$ ameliorates the glial activation and VEGF expression in the retina of the type 2 diabetic murine model. As shown in Figure 7, tofogliflozin decreased the overexpression of GFAP (Figure 7A) and VEGF (Figure 7B) in the $\mathrm{db} / \mathrm{db}$ mouse retina. There were significant increases in fluorescein intensities of GFAP and VEGF in the untreated $\mathrm{db} / \mathrm{db}$ mice, compared with non-diabetic $\mathrm{db} / \mathrm{m}$ mice, but the increased intensities of both GFAP (Figure 7A) and VEGF (Figure 7B) were significantly reduced in SGLT2i-treated mice retina. 
$\begin{array}{ll}\text { A. GFAP } & \text { (a) } d b / m\end{array}$

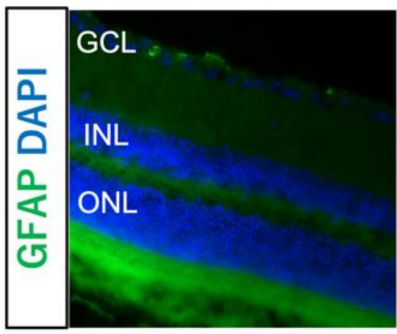

B. VEGF

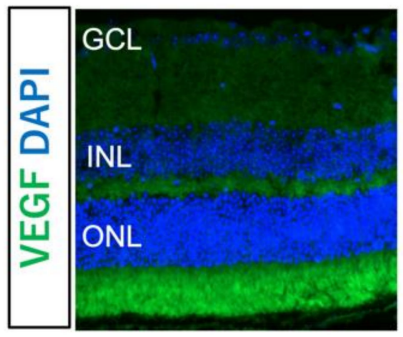

(b) db/db +Normal feed

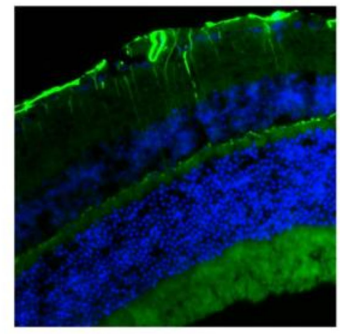

(b) $\mathrm{db} / \mathrm{db}+$ Normal feed

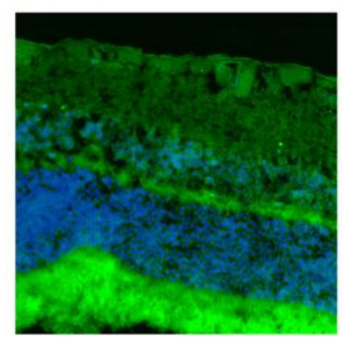

(c) $\mathrm{db} / \mathrm{db}+$ Tofogliflozine

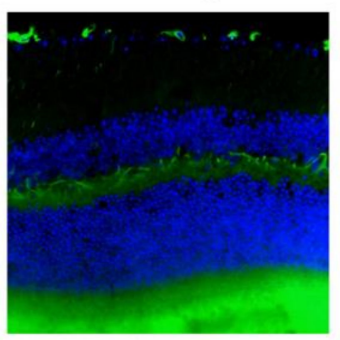

(c) $d b / d b+$ Tofogliflozine

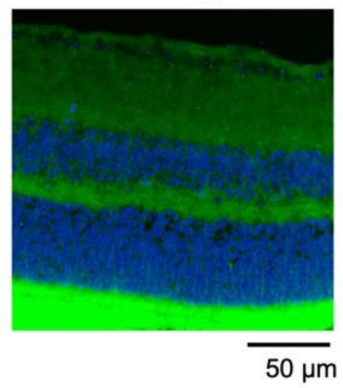

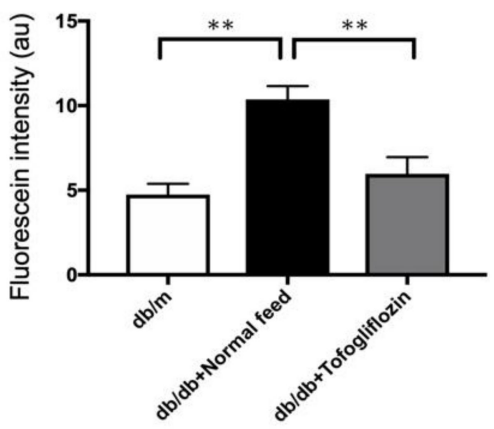

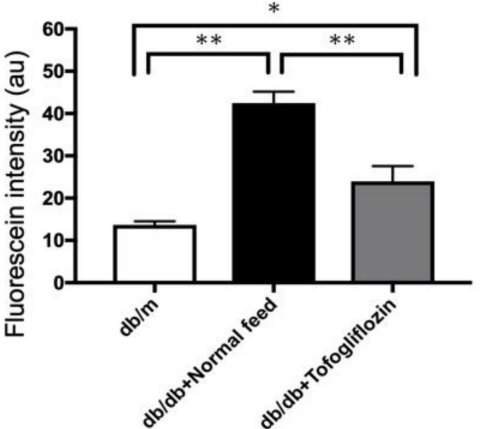

Figure 7. Effect of SGLT2i tofogliflozin on the glial activation (A) and production of VEGF (B). Comparison of GFAP immunofluorescence (green) between representative samples from in $\mathrm{db} / \mathrm{m}$ (a) and untreated (b) or SGLT2i-treated (c) db/db mice at 14 weeks. In the diabetic retina with normal feed (b), the end-feet of the Müller cells show abundant GFAP immunofluorescence, and the radial processes are stained intensely throughout both the inner and outer retina. In the diabetic retina with tofogliflozin (b), the immunofluorescence of both GFAP and VEGF was reduced and comparable with that in $\mathrm{db} / \mathrm{m}$ control mice (a). There were significant differences in the fluorescein intensities of GFAP $(p=0.005)$ and VEGF $(p<0.0001)$ among the three groups by one-way ANOVA. There were significant increases in intensities of GFAP and VEGF in the untreated $\mathrm{db} / \mathrm{db}$ mice $(n=6)$, compared with the non-diabetic $\mathrm{db} / \mathrm{m}$ mice $(n=5)$. These increases were significantly ameliorated in the tofogliflozin-treated murine retina $(n=6)$. ${ }^{*} p<0.05,{ }^{* *} p<0.01$. Nuclei were labeled with DAPI (blue). ONL, outer nuclear layer; INL, inner nuclear layer; GCL, ganglion cell layer. Scale bar $=50 \mu \mathrm{m}$.

\section{Discussion}

In the current study, we confirmed that the long-term administration of SGLT2 $i$ tofogliflozin decreased the blood glucose level, compared with untreated $\mathrm{db} / \mathrm{db}$ mice (Table 1). Persistent hyperglycemia drives complex biochemical and molecular changes that lead to oxidative stress/inflammation, vascular endothelial cell damage, capillary ischemia, and tissue hypoxia [10]. Indeed, we recently confirmed that RBF dysregulation in response to flicker stimulation, which indicates impaired neurovascular coupling in the retina [11], occurred from 8 to 20 weeks of age in $\mathrm{db} / \mathrm{db}$ mice before the apparent neuronal abnormalities, which can be detected by ERG [9]. As pericyte loss was observed at 24 weeks of age but not 12 weeks of age in $\mathrm{db} / \mathrm{db}$ mice [12], the impaired neurovascular coupling in the retina may be the earliest event in the diabetic retina. In the current study, glycemic control by the SGLT2i tofogliflozin ameliorated the impaired blood flow response to flicker stimulation with the improvement of glycemic control, suggesting that glucotoxicity to the retinal neurovascular unit can be a primary risk factor for impaired neurovascular coupling in the retina. Our findings seem to support the recent American Diabetic Association statement that DR should be reclassified from a microvascular complication to a neurovascular complication since damage to the specialized neurons in the eye plays an important role in the development and progression of DR [13]. 
Previous clinical studies have shown that the SGLT2i treatment decreases systemic BP $[14,15]$. However, there were no significant changes in systemic BP, IOP, or OPP in either untreated or SGLT2i-treated mice during the follow-up period in the current study (Figure 1). In contrast, a previous animal study also reported that empagliflozin ameliorates kidney injury in type 2 diabetic female mice by promoting glycosuria without any changes in systemic BP [16]. Their findings seem to be comparable to ours.

We used the SGLT2 inhibitor tofogliflozin to restore glycemic control in $\mathrm{db} / \mathrm{db}$ mice. SGLT2 inhibitors are the latest class of anti-diabetic medications. In addition, a recent clinical study reported that another SGLT2i, empagliflozin, reduces the risk of major adverse cardiovascular events [17] and reduces the progression of kidney diseases [18], suggesting that SGLT2is may have a beneficial effect on both macrovascular and microvascular complications in diabetic patients. Indeed, a recent real-world cohort study showed that SGLT2is may be associated with a lower risk of DR, compared with DPP4is [5]. In addition, the treatment of type 2 diabetic patients with an SGLT2i slowed the progression of DR in comparison with sulfonylurea, which occurs independently of its effect on glycemic control [4]. Taken together, a large-scale prospective clinical study to examine the effect of SGLT2i on the prevention of DR is warranted.

Eid et al., reported that the long-term administration of empagliflozin for 10 weeks from 6 to 16 weeks of age did not ameliorate any microvascular complications in $\mathrm{db} / \mathrm{db}$ mice [19]. In their study, DR was assessed by the measurement of cleaved apoptotic DNA in the retina using an ELISA. We confirmed that the RBF abnormalities occurred at 8 weeks of age in $\mathrm{db} / \mathrm{db}$ mice, which preceded the retinal neuronal dysfunction evaluated by ERG. Although different types of SGLT2is were used in the two studies, RBF provocation of systemic hyperoxia and flicker stimulation may be useful as a biomarker for the detection of early retinal dysfunction.

A previous study showed that sodium-dependent glucose uptake is present in bovine retinal pericytes and human retinal endothelial cells [20]. In contrast, histologic studies assessing the expression of SGLT2 directly in human retinas have not been reported widely in the literature [21]. In our preliminary study, we could not find any expression of SGLT2 in the $\mathrm{db} / \mathrm{db}$ mouse retina in Supplementary Materials (Figure S1). Therefore, we speculate that the glucose-lowering effect induced by the SGLT2i tofogliflozin may ameliorate the diabetes-induced impairment of RBF regulation in response to flicker stimulation and systemic hyperoxia. If this is the case, the improvement in the neurovascular function in the retina of $\mathrm{db} / \mathrm{db}$ mice may be associated with glycemic control rather than the inhibition of SGLT2 in the retina.

We found that the long-term administration of tofogliflozin decreased the GFAP expression in the $\mathrm{db} / \mathrm{db}$ mouse retina (Figure 7). Our result seems to be comparable with an increasing number of studies that show that diabetes is associated with reactive Müller cell gliosis [22,23], which is characterized by increased levels of GFAP. As the retinal glial cells play important roles in the regulation of RBF in response to systemic hyperoxia [24] and flicker stimulation [25], the beneficial effect of SGLT2i on retinal glial cells may contribute to the recovery of the diabetes-induced retinal dysfunction via improved glycemic control. In the diabetic brain, the SGLT2i empagliflozin prevented glial activation in the neurovascular unit, suggesting that empagliflozin may provide neuroprotection in the diabetic brain via the protection of glial cells [26]. In addition, the non-selective SGLT inhibitor phlorizin ameliorates the endothelial dysfunction link with the activation of the PI3K/AKT/eNOS signaling pathway and augmentation of the release of nitric oxide (NO) [27], and SGLT2 $i$ EMPA, and DAPA restores NO bioavailability by inhibiting reactive oxygen species (ROS) generation [28]. It is difficult to confirm the exact mechanism of neurovascular coupling in the retina in response to flicker stimulation; glial activation and/or the restored impaired endothelial function may be involved in the restoration of RBF regulation in response to the systemic hyperoxia and flicker stimulation.

In the present study, we first observed that the increased VEGF expression in the retina decreased with long-term SGLT2i treatment in diabetic mice at 14 weeks of age 
(Figure 7). Amin et al. [29] revealed that VEGF immunopositivity was present in eyes with no retinal vascular anatomic abnormality in retinal digest preparations from diabetic patients with non-proliferative DR. Their results are comparable to our findings. Wang et al., clearly showed that the conditional VEGF knockout (KO) in Müller cells of diabetic mice exhibited significantly reduced leukostasis, expression of inflammatory biomarkers, and vascular leakage, compared with STZ-induced diabetic control mice. [30] These results suggest that the glucotoxicity-related VEGF overexpression may be produced from the activated Müller cells in the diabetic retina [29]. In addition, $\mathrm{Mu}$ et al., reported that the levels of VEGF mRNA and protein increased in cultured Müller cells under high glucose concentrations [31]. As persistent gliosis leads to the loss of normal Müller glial cell function and subsequent loss of retinal neurons [32], our data suggest that glucotoxicityinduced gliosis of Müller cells, which can be detected the increased GFAP expression, may lead to the overproduction of VEGF in the diabetic retina as an early event in the diabetic retina. Moreover, we believe that the long-term administration of the SGLT2i can ameliorate the retinal neurovascular coupling by reducing the activation of Müller cells and the overexpression of VEGF by lowering blood glucose levels.

Clermont et al., reported that acute glycemic control by primary intervention using insulin pumps caused the normalization of the RBF in type 1 STZ-induced diabetic rats [33], suggesting that glucotoxicity may be involved with the impaired RBF in diabetic animals. However, no studies have investigated whether long-term glycemic control ameliorates the RBF dysregulation in diabetic animals. In the present study, we reported, for the first time, that long-term glycemic control by SGLT2i results in the improvement of the RBF response to flicker stimulation and systemic hyperoxia in type 2 diabetic mice from 8 to 14 weeks of age. In addition, a long-term glycemic control by SGLT2i reduced the delay of the implicit time of the oscillatory potentials from 12 to 14 weeks of age in diabetic mice. The current results suggest that glycemic control is very important for maintaining a normal retinal neuronal and vascular function in the early stage of diabetes.

Although some recent clinical studies have reported the beneficial effect of SGLT2i on DR [4,5] and diabetic macular edema [6,7], the EMPA-REG outcome trial could not find a beneficial effect of empagliflozin on DR [8]. Eid et al., reported that the long-term administration of empagliflozin for 10 weeks from 6 to 16 weeks of age did not ameliorate any microvascular complications in $\mathrm{db} / \mathrm{db}$ mice [19]. In their study, they evaluated cleaved apoptotic DNA using an ELISA to assess "DR". In the current study, we confirmed that retinal functional abnormalities occurred at 8 weeks of age in $\mathrm{db} / \mathrm{db}$ mice. A clinical trial is needed to investigate whether the SGLT2is may have a beneficial effect on DR, especially at the early stage of DR, by evaluating the abnormalities of the RBF regulation in response to systemic hyperoxia and flicker stimulation, as we conducted in our study. Glycemic control by SGLT2i may contribute to the prevention of diabetic macular edema and DR.

We previously confirmed that the RBF response to systemic hyperoxia and flicker stimulation remains stable from 8 to 20 weeks of age in $\mathrm{db} / \mathrm{m}$ non-diabetic control mice [9]. Due to the animal welfare criteria in our institution, we performed only one RBF and ERG experiment at 14 weeks of age in $\mathrm{db} / \mathrm{m}$ mice. We found that the maximal responses of the $\mathrm{RBF}$ in response to hyperoxia and flicker stimulation in $\mathrm{db} / \mathrm{db}$ mice with tofogliflozin had not fully recovered to the same levels as those in non-diabetic $\mathrm{db} / \mathrm{m}$ mice at 14 weeks of age (Figure 6), but there were no significant differences in either response between the groups, suggesting that tofogliflozin can improve the normal responses of the RBF to systemic hyperoxia and flicker stimulation. Further study is warranted to confirm the effect of supplementation on the prevention of DR in a diabetic animal model, which expresses DR, or in human patients with diabetes.

The present study had some limitations. First, we could not exclude the possibility that a direct effect of the inhibition of SGLT2 in the retina, which was reported in bovine retinal pericytes, may have had some effects on our results in accordance with the glycemic control by the systemic administration of SGLT2i in the current study. Second, we did not confirm whether SGLT2i tofogliflozin prevents the pathological changes of DR. As it 
has been reported that the pericyte loss occurred at 24 weeks of age in $\mathrm{db} / \mathrm{db}$ mice [12], a longitudinal observational study with a longer follow-up period is needed.

In conclusion, we found that long-term glycemic control with SGLT2i tofogliflozin significantly ameliorated the glial activation and VEGF expression in the retina and improved the RBF regulation dysfunction and neural retinal dysfunction in type 2 diabetic mice. These results suggest that the SGLT2i may contribute to the prevention of DR and diabetic macular edema.

\section{Materials and Methods}

\subsection{Animal Preparation}

The Nihon University Ethical Committee approved the animal experiments, which were carried out according to the tenets of the Association of Research in Vision and Ophthalmology. We also confirmed that this survey was conducted in accordance with the ARRIVE guidelines (https:/ /arriveguidelines.org (accessed on 13 December 2021)) Five-week-old male C57BL/KsJ-db/db mice (BKS.Cg-Dock7m +/+ Leprdb/J; $n=12$ ) and 13-week-old male $\mathrm{db} / \mathrm{m}$ (non-diabetic congenic littermates, $n=5$ ) control mice were obtained from Charles River Laboratories Japan Co., Ltd. (Yokohama, Japan). They were divided into a placebo group $(n=6)$, with ad libitum access to normal chow, and a treatment group $(n=6)$, with ad libitum access to chow containing tofogliflozin hydrate. We obtained tofogliflozin from Kowa Co., Ltd. (Tokyo, Japan). Only male db/db mice were used because diabetes is more severe in male $\mathrm{db} / \mathrm{db}$ mice than in females [34]. Blood glucose levels were measured from the tail vein (glucose assay kit; Abbott Laboratories, Abbott Park, IL, USA). Mice were housed in a temperature-controlled room, with a $12 \mathrm{~h}$ light-dark cycle and ad libitum access to food and water. Throughout the experiment, mice were anesthetized with $2 \%$ isoflurane (Pfizer, Tokyo, Japan) continuously inhaled at a flow rate of $1.5 \mathrm{~L} / \mathrm{min}$. The pupil was dilated with $0.5 \%$ tropicamide (Santen Pharmaceutical Co., Ltd., Osaka, Japan). The rectal temperature was measured and a heated blanket was used to maintain the temperature between 37 and $38^{\circ} \mathrm{C}$.

\subsection{Chemicals and Systemic Administration Protocol}

Tofogliflozin was kindly provided by Kowa Company, Ltd. The $\mathrm{db} / \mathrm{db}$ mice were randomly allocated to either the untreated group $(n=6)$ or the treatment group $(n=6)$. $\mathrm{The} \mathrm{db} / \mathrm{db}$ mice in both groups had ad libitum access to solidified regular food. In the treatment group, the solidified food was mixed with $0.004 \%$ tofogliflozin $(5 \mathrm{mg} / \mathrm{kg} /$ day $)$ at the same dose as reported in the previous study [35].

\subsection{Systemic BP and IOP Measurements}

Systemic BP and IOP were measured $30 \mathrm{~min}$ after the induction of anesthesia. Tail systolic BP (SBP) and diastolic BP (DBP) were measured using an automatic sphygmomanometer (THC-31, Softron, Tokyo, Japan). IOP was measured by a handheld tonometer (TonolabTV02, ME Technical, Tokyo, Japan). The mean arterial pressure (MABP) was derived from the standard formula: $\mathrm{MABP}=\mathrm{DBP}+(\mathrm{SBP}-\mathrm{DBP}) / 3$.

The OPP was calculated using the following formula for an experimental animal in the prone position [36]: OPP = MABP - IOP.

\subsection{RBF Measurement}

RBF was measured using the LSFG Micro system (Softcare Co., Ltd., Fukutsu, Japan), as previously described [9]. In brief, the LSFG-Micro system includes a standard chargecoupling device camera (700 $\times 480$ pixels) and a diode laser $(830 \mathrm{~nm}$ wavelength) mounted on a stereomicroscope (SZ61TR, Olympus Corporation, Tokyo, Japan). The mean blur rate (MBR), a relative indicator of blood flow velocity, is generated from the speckle pattern blur formed by the backscattered light of a coherent laser due to the movement of blood cells. The MBR obtained from the area around the optic nerve head $(\mathrm{ONH})$ and its vascular area reflects the entire retinal circulation and is used as an indicator of RBF [36]. In this 
study, the MBR was obtained as previously described [9]. The mouse was placed on the stand with the left eye facing up. A drop of viscoelastic material was dropped and the cover glass was gently placed on the left cornea. The ONH margin was shown by manually placing a rubber $\mathrm{O}$ ring (diameter: $1.37 \mathrm{~mm}$ ) on the $\mathrm{ONH}$ fundus image. The MBR was continuously acquired at 30 frames/second within the O-ring area. The average MBR was analyzed using the LSFG analyzer software program (version 3.2.19.0, Softcare Co., Ltd., Fukutsu, Japan).

\subsection{Induction of Systemic Hyperoxia}

Systemic hyperoxia was induced by the inhalation of $100 \%$ oxygen over $10 \mathrm{~min}$, as explained in our previous study [36]. The baseline value was determined as the average of three consecutive flow measurements taken at $1 \mathrm{~min}$ intervals for $3 \mathrm{~min}$. To determine the onset of hyperoxia, RBF measurements were taken every minute for 20 min during hyperoxia (10 min of stimulation) and after the end of hyperoxia (10 min of recovery) $[9,36]$.

\subsection{Flicker Light Stimulation}

A $12 \mathrm{~Hz}$ flicker stimulation was used because this frequency causes the maximal RBF response in mice, as explained in our previous study [36]. The ambient light was reduced to less than 1 lux before inducing flicker stimulation. As previously reported, mice were dark adapted for $2 \mathrm{~h}$, and the light intensity of flicker stimulation was 30 lux in rod-dominant mouse retinas [9,36]. RBF was measured at $20 \mathrm{~s}$ intervals, with both $3 \mathrm{~min}$ flickers. Stimulation and recovery lasted for $3 \mathrm{~min}$. Baseline values were calculated using the average of three consecutive flow measurements taken at $1 \mathrm{~min}$ ( $20 \mathrm{~s}$ intervals) before the start of light flicker stimulation.

\subsection{ERG Recording}

Before ERG, mice were dark adapted for at least $12 \mathrm{~h}$ before being transferred to a room with dim red light. Full-field ERG was recorded using PuREC (Mayo, Inazawa, Japan) under systemic anesthesia with isoflurane. The ground electrode was attached to the tail, the reference electrode was attached to the mouth, and the corneal electrodes were placed on the corneal surface. As previously reported, we achieved maximal responses for both cones and rods using a 3.0 candela $/ \mathrm{m}^{2}$ flash [36]. OPs are small radio frequency vibration wavelets superimposed on the upper limb of the b-wave. OP wavelets were labeled sequentially as OP1-OP3, starting with the first positive peak detected. The OP amplitude (positive amplitude of the peak-negative amplitude of the peak of the previous peak), and implicit time were measured. The OP amplitude was calculated by adding the first three positive wavelets and expressed as the $\Sigma \mathrm{OP}$ amplitude.

\subsection{Experimental Protocols}

At 6 weeks of age, before the administration of tofogliflozin, we measured the body weight and blood glucose. The following protocol was performed in each animal for 3 consecutive days, every 2 weeks from 8 to 14 weeks of age, for longitudinal assessment of $\mathrm{RBF}$ regulation and the neural function: The systemic hyperoxia reaction of RBF occurred on the first day; the following day, systemic BP, IOP, and OPP were not altered by the reaction to high oxygen or light flicker stimulation. On the third day, we recorded the ERG. An independent masked observer (A.K.) performed all data calculations and statistical analysis.

\subsection{Immunohistochemistry}

After the measurement at 14 weeks of age, all animals, including untreated $(n=6)$ and SGLT2i-treated $(n=6) \mathrm{db} / \mathrm{db}$ and $\mathrm{db} / \mathrm{m}(n=5)$ mice, were euthanized, and the eyeballs were excised, as follows: Sternotomy was performed under systemic anesthesia with $3 \%$ isoflurane; normal saline was perfused into the left ventricle to wash out the circulating blood, immediately, followed by perfusion of $4 \%$ paraformaldehyde (PFA), and the eyeballs were enucleated. The eyeballs were stored in $4 \%$ PFA at $4{ }^{\circ} \mathrm{C}$ overnight; then, after a 
couple of washings in phosphate-buffered saline, they were embedded in Tissue-Tek OCT Compound (Sakura Finetek Japan, Tokyo, Japan) and stored at $-80{ }^{\circ} \mathrm{C}$ until analysis.

A total of 10 micrometer-thick sections were prepared with a cryostat (HM505, Microm, Walldorf, Germany). Sections were stained with GFAP (ready to use, Dako, Glostrup, Denmark), ionized calcium-binding adapter molecule 1 (iba-1; dilution, 1:200; Wako Pure Chemical Industries Ltd., Osaka, Japan), and VEGF (1:100, Sigma-Aldrich, St. Louis, MO, USA) overnight at $4{ }^{\circ} \mathrm{C}$. Next, they were incubated with secondary donkey anti-rabbit IgG $(\mathrm{H}+\mathrm{L})$ Alexa Fluor 488 (dilution, 1:400; Thermo Fisher Scientific, Waltham, MA, USA) for $2 \mathrm{~h}$ at room temperature. Immunostaining was developed with a Histofine Simple Stain PO (M) Kit (Nichirei, Tokyo, Japan) per the instruction manual. Immunofluorescent images were obtained by a FluoView 1000 confocal microscope (Olympus, Tokyo, Japan) and a BZ-9000 microscope (Keyence, Osaka, Japan). The fluorescein intensities were calculated using ImageJ software.

\subsection{Statistical Analysis}

Data were expressed as the mean \pm standard error of the mean. The changes in RBF were calculated as percentage changes from baseline. The $n$ value indicates the number of animals studied. The Kolmogorov-Smirnov test was used to assess the normality of data distribution. Differences among groups and time points were assessed using a one-way or two-way repeated-measures ANOVA, as appropriate, followed by Dunnett's test or the Holm-Sidak test. Prism 9 (GraphPad Software, San Diego, CA, USA) was used to perform all statistical analyses. $p$-values of $<0.05$ were considered statistically significant.

Supplementary Materials: Supplementary materials can be found at https://www.mdpi.com/ article/10.3390/ijms23031362/s1.

Author Contributions: Conceptualization, T.N., H.Y. and A.K.; methodology, T.N., H.Y. and S.K.; validation, T.N.; formal analysis, J.H.; investigation, J.H., M.W.; resources, T.N., S.Y.; writing—original draft preparation, T.N. and J.H.; writing—review and editing, A.K., H.Y. and T.N.; supervision, S.Y. All authors have read and agreed to the published version of the manuscript.

Funding: This study was supported by a Grant-in-Aid for Scientific Research (C) 26861430 from the Ministry of Education, Science, and Culture, Tokyo, Japan, a grant from the Uehara Memorial Foundation, and a research fund from Kowa Co. Ltd. (to T.N.).

Institutional Review Board Statement: Protocols using animas were permitted by the Nihon University Ethical Committee (Approved number \#AP20MED014-2/2020).

Informed Consent Statement: Not applicable.

Data Availability Statement: The data presented in this study are available on request from the corresponding author.

Acknowledgments: We thank Akiko Tomioka for her excellent technical assistance. We also thank Kowa Company to provide tofogliflozin.

Conflicts of Interest: The authors declare no conflict of interest.

\section{References}

1. Bourne, R.R.; Stevens, G.A.; White, R.A.; Smith, J.L.; Flaxman, S.R.; Price, H.; Jonas, J.B.; Keeffe, J.; Leasher, J.; Naidoo, K.; et al. Causes of vision loss worldwide, 1990-2010: A systematic analysis. Lancet Glob. Health 2013, 1, e339-e349. [CrossRef]

2. Kawasaki, R.; Tanaka, S.; Tanaka, S.; Yamamoto, T.; Sone, H.; Ohashi, Y.; Akanuma, Y.; Yamada, N.; Yamashita, H.; Japan Diabetes Complications Study Group. Incidence and progression of diabetic retinopathy in Japanese adults with type 2 diabetes: 8 year follow-up study of the Japan Diabetes Complications Study (JDCS). Diabetologia 2011, 54, 2288-2294. [CrossRef] [PubMed]

3. Devineni, D.; Curtin, C.R.; Polidori, D.; Gutierrez, M.J.; Murphy, J.; Rusch, S.; Rothenberg, P.L. Pharmacokinetics and pharmacodynamics of canagliflozin, a sodium glucose co-transporter 2 inhibitor, in subjects with type 2 diabetes mellitus. J. Clin. Pharmacol. 2013, 53, 601-610. [CrossRef] [PubMed]

4. Cho, E.H.; Park, S.J; Han, S.; Song, J.H.; Lee, K.; Chung, Y.R. Potent Oral Hypoglycemic Agents for Microvascular Complication: Sodium-Glucose Cotransporter 2 Inhibitors for Diabetic Retinopathy. J. Diabetes Res. 2018, 2018, 6807219. [CrossRef] 
5. Chung, Y.R.; Ha, K.H.; Lee, K.; Kim, D.J. Effects of sodium-glucose cotransporter-2 inhibitors and dipeptidyl peptidase-4 inhibitors on diabetic retinopathy and its progression: A real-world Korean study. PLoS ONE 2019, 14, e0224549. [CrossRef]

6. Su, Y.C.; Shao, S.C.; Lai, E.C.; Lee, C.N.; Hung, M.J.; Lai, C.C.; Hsu, S.M.; Hung, J.H. Risk of diabetic macular oedema with sodium-glucose cotransporter-2 inhibitors in type 2 diabetes patients: A multi-institutional cohort study in Taiwan. Diabetes Obes. Metab. 2021, 23, 2067-2076. [CrossRef]

7. Takatsuna, Y.; Ishibashi, R.; Tatsumi, T.; Koshizaka, M.; Baba, T.; Yamamoto, S.; Yokote, K. Sodium-Glucose Cotransporter 2 Inhibitors Improve Chronic Diabetic Macular Edema. Case Rep. Ophthalmol. Med. 2020, 2020, 8867079. [CrossRef]

8. Inzucchi, S.E.; Wanner, C.; Hehnke, U.; Zwiener, I.; Kaspers, S.; Clark, D.; George, J.T.; Zinman, B. Retinopathy Outcomes with Empagliflozin Versus Placebo in the EMPA-REG OUTCOME Trial. Diabetes Care 2019, 42, e53-e55. [CrossRef]

9. Hanaguri, J.; Yokota, H.; Watanabe, M.; Yamagami, S.; Kushiyama, A.; Kuo, L.; Nagaoka, T. Retinal blood flow dysregulation precedes neural retinal dysfunction in type 2 diabetic mice. Sci. Rep. 2021, 11, 18401. [CrossRef]

10. Brownlee, M. The pathobiology of diabetic complications: A unifying mechanism. Diabetes 2005, 54, 1615-1625. [CrossRef]

11. Riva, C.E.; Harino, S.; Shonat, R.D.; Petrig, B.L. Flicker evoked increase in optic nerve head blood flow in anesthetized cats. Neurosci. Lett. 1991, 128, 291-296. [CrossRef]

12. Midena, E.; Segato, T.; Radin, S.; di Giorgio, G.; Meneghini, F.; Piermarocchi, S.; Belloni, A.S. Studies on the retina of the diabetic $\mathrm{db} / \mathrm{db}$ mouse. I. Endothelial cell-pericyte ratio. Ophthalmic Res. 1989, 21, 106-111. [CrossRef]

13. Solomon, S.D.; Chew, E.; Duh, E.J.; Sobrin, L.; Sun, J.K.; VanderBeek, B.L.; Wykoff, C.C.; Gardner, T.W. Diabetic Retinopathy: A Position Statement by the American Diabetes Association. Diabetes Care 2017, 40, 412-418. [CrossRef]

14. Scheen, A.J. Sodium-glucose cotransporter type 2 inhibitors for the treatment of type 2 diabetes mellitus. Nat. Rev. Endocrinol. 2020, 16, 556-577. [CrossRef]

15. Tsapas, A.; Karagiannis, T.; Kakotrichi, P.; Avgerinos, I.; Mantsiou, C.; Tousinas, G.; Manolopoulos, A.; Liakos, A.; Malandris, K.; Matthews, D.R.; et al. Comparative efficacy of glucose-lowering medications on body weight and blood pressure in patients with type 2 diabetes: A systematic review and network meta-analysis. Diabetes Obes. Metab. 2021, 23, 2116-2124. [CrossRef]

16. Aroor, A.R.; Das, N.A.; Carpenter, A.J.; Habibi, J.; Jia, G.; Ramirez-Perez, F.I.; Martinez-Lemus, L.; Manrique-Acevedo, C.M.; Hayden, M.R.; Duta, C.; et al. Glycemic control by the SGLT2 inhibitor empagliflozin decreases aortic stiffness, renal resistivity index and kidney injury. Cardiovasc. Diabetol. 2018, 17, 108. [CrossRef]

17. Zinman, B.; Wanner, C.; Lachin, J.M.; Fitchett, D.; Bluhmki, E.; Hantel, S.; Mattheus, M.; Devins, T.; Johansen, O.E.; Woerle, H.J.; et al. Empagliflozin, Cardiovascular Outcomes, and Mortality in Type 2 Diabetes. N. Engl. J. Med. 2015, 373, 2117-2128. [CrossRef]

18. Wanner, C.; Inzucchi, S.E.; Lachin, J.M.; Fitchett, D.; von Eynatten, M.; Mattheus, M.; Johansen, O.E.; Woerle, H.J.; Broedl, U.C.; Zinman, B.; et al. Empagliflozin and Progression of Kidney Disease in Type 2 Diabetes. N. Engl. J. Med. 2016, 375, 323-334. [CrossRef]

19. Eid, S.A.; O’Brien, P.D.; Hinder, L.M.; Hayes, J.M.; Mendelson, F.E.; Zhang, H.; Zeng, L.; Kretzler, K.; Narayanan, S.; Abcouwer, S.F.; et al. Differential Effects of Empagliflozin on Microvascular Complications in Murine Models of Type 1 and Type 2 Diabetes. Biology 2020, 9, 347. [CrossRef]

20. Wakisaka, M.; Kitazono, T.; Kato, M.; Nakamura, U.; Yoshioka, M.; Uchizono, Y.; Yoshinari, M. Sodium-coupled glucose transporter as a functional glucose sensor of retinal microvascular circulation. Circ. Res. 2001, 88, 1183-1188. [CrossRef]

21. Lahoti, S.; Nashawi, M.; Sheikh, O.; Massop, D.; Mir, M.; Chilton, R. Sodium-glucose co-transporter 2 inhibitors and diabetic retinopathy: Insights into preservation of sight and looking beyond. Cardiovasc. Endocrinol. Metab. 2021, 10, 3-13. [CrossRef]

22. Coughlin, B.A.; Feenstra, D.J.; Mohr, S. Muller cells and diabetic retinopathy. Vis. Res. 2017, 139, 93-100. [CrossRef]

23. Trueblood, K.E.; Mohr, S.; Dubyak, G.R. Purinergic regulation of high-glucose-induced caspase-1 activation in the rat retinal Muller cell line rMC-1. Am. J. Physiol. Cell Physiol. 2011, 301, C1213-C1223. [CrossRef] [PubMed]

24. Song, Y.; Nagaoka, T.; Yoshioka, T.; Ono, S.; Wada, T.; Nakabayashi, S.; Tani, T.; Yoshida, A. Glial endothelin-1 regulates retinal blood flow during hyperoxia in cats. Investig. Ophthalmol. Vis. Sci. 2016, 57, 4962-4969. [CrossRef]

25. Song, Y.; Nagaoka, T.; Yoshioka, T.; Nakabayashi, S.; Tani, T.; Yoshida, A. Role of glial cells in regulating retinal blood flow during flicker-induced hyperemia in cats. Investig. Ophthalmol. Vis. Sci. 2015, 56, 7551-7559. [CrossRef]

26. Hayden, M.R.; Grant, D.G.; Aroor, A.R.; DeMarco, V.G. Empagliflozin Ameliorates Type 2 Diabetes-Induced Ultrastructural Remodeling of the Neurovascular Unit and Neuroglia in the Female db/db Mouse. Brain Sci. 2019, 9, 83. [CrossRef]

27. Li, C.Y.; Wang, L.X.; Dong, S.S.; Hong, Y.; Zhou, X.H.; Zheng, W.W.; Zheng, C. Phlorizin Exerts Direct Protective Effects on Palmitic Acid (PA)-Induced Endothelial Dysfunction by Activating the PI3K/AKT/eNOS Signaling Pathway and Increasing the Levels of Nitric Oxide (NO). Med. Sci. Monit. Basic Res. 2018, 24, 1-9. [CrossRef]

28. Uthman, L.; Homayr, A.; Juni, R.P.; Spin, E.L.; Kerindongo, R.; Boomsma, M.; Hollmann, M.W.; Preckel, B.; Koolwijk, P.; van Hinsbergh, V.W.M.; et al. Empagliflozin and Dapagliflozin Reduce ROS Generation and Restore NO Bioavailability in Tumor Necrosis Factor alpha-Stimulated Human Coronary Arterial Endothelial Cells. Cell Physiol. Biochem. 2019, 53, 865-886. [PubMed]

29. Amin, R.H.; Frank, R.N.; Kennedy, A.; Eliott, D.; Puklin, J.E.; Abrams, G.W. Vascular endothelial growth factor is present in glial cells of the retina and optic nerve of human subjects with nonproliferative diabetic retinopathy. Investig. Ophthalmol. Vis. Sci. $1997,38,36-47$.

30. Wang, J.; Xu, X.; Elliott, M.H.; Zhu, M.; Le, Y.Z. Muller cell-derived VEGF is essential for diabetes-induced retinal inflammation and vascular leakage. Diabetes 2010, 59, 2297-2305. [CrossRef] 
31. Mu, H.; Zhang, X.M.; Liu, J.J.; Dong, L.; Feng, Z.L. Effect of high glucose concentration on VEGF and PEDF expression in cultured retinal Muller cells. Mol. Biol. Rep. 2009, 36, 2147-2151. [CrossRef] [PubMed]

32. Goldman, D. Muller glial cell reprogramming and retina regeneration. Nat. Rev. Neurosci. 2014, 15, 431-442. [CrossRef] [PubMed]

33. Clermont, A.C.; Brittis, M.; Shiba, T.; McGovern, T.; King, G.L.; Bursell, S.E. Normalization of retinal blood flow in diabetic rats with primary intervention using insulin pumps. Investig. Ophthalmol. Vis. Sci. 1994, 35, 981-990.

34. Kitada, M.; Ogura, Y.; Koya, D. Rodent models of diabetic nephropathy: Their utility and limitations. Int. J. Nephrol. Renovasc. Dis. 2016, 9, 279-290. [CrossRef]

35. Ishibashi, Y.; Matsui, T.; Yamagishi, S.I. Tofogliflozin, a selective inhibitor of sodium-glucose cotransporter 2, suppresses renal damage in KKAy/Ta mice, obese and type 2 diabetic animals. Diabetes Vasc. Dis. Res. 2016, 13, 438-441. [CrossRef]

36. Hanaguri, J.; Yokota, H.; Watanabe, M.; Kuo, L.; Yamagami, S.; Nagaoka, T. Longitudinal stability of retinal blood flow regulation in response to flicker stimulation and systemic hyperoxia in mice assessed with laser speckle flowgraphy. Sci. Rep. 2020, 10, 19796. [CrossRef] 